\title{
Perception of Cancer Risk and Its Associated Risk Factors among Young Iraqis living in Baghdad
}

\author{
Harith Alaa, Shamsul Azhar Shah*
}

\begin{abstract}
Cancer is responsible for substantial burden on communities and more specifically on less developed countries. The incidence of cancer is on the rise due to population growth and aging, also due to increment of the risk factors such as smoking, increasing weight, low physical activity associated with adoption of western lifestyle. Around 14 million cases of new cancer and 8 million deaths from cancer is estimated to occur by 2012. This cross-sectional study was conducted in Baghdad from June 2016 to October 2016. Participants were selected according to our inclusion criteria, namely aged between 18 to 40 years and not being diagnosed with any chronic diseases. Those who fulfilled the inclusion criteria were 700 participants who completed the questionnaire. Results showed that most of our participants had low perceived susceptibility to cancer risk (62.4\%), low perceived severity $(59.8 \%)$, but good perceived benefits of screening $(56.6 \%)$. Hierarchal linear regression analysis showed that sociodemographic factors of gender, marital status, and education level were statistically significant. Moreover, factors of health behaviour such as practice towards health and preventive behaviour were associated with the outcome. Finally, treatment control and emotional factors were mostly predicting the outcome. Perceived susceptibility to cancer along with its psychological factors and behaviour were important contributors to self-perceived health in this study. Hence there is association between perception and future morbidity and mortality, thus it is crucial for public health policy. Comprehensive health programs that include health promotion campaigns and proper health care services that deals with secondary prevention.
\end{abstract}

Keywords: Cancer- perception- young adults- Iraq

Asian Pac J Cancer Prev, 20 (8), 2339-2343

\section{Introduction}

Cancer is the second leading cause of death globally, accounting for an estimated 9.6 million deaths in 2018. Worldwide, about 1 in 6 deaths is due to cancer. Approximately, $70 \%$ of deaths from cancer occur in low- and middle-income countries. Around one third of deaths from cancer are due to the 5 leading behavioural and dietary risks: high body mass index, low fruit and vegetable intake, lack of physical activity, tobacco use, and alcohol use. Cancer disease is responsible for substantial burden on communities and more specifically on less developing countries. Its incidence is on the rise due to population growth and aging, and also due to increment of the risk factors such as smoking, excess body weight, low physical activity that are associated with western lifestyle adoption. It is estimated that around 14 million cases of new cancer and about 8 million cancer deaths will be found in 2012 (Torre et al., 2015). On the other hand, around $50 \%$ of cancer diseases can be prevented through avoiding cancerous risk factors and providing proper preventive strategies. It was found that the first three cancers for men were lung, prostate, and colon cancer with prevalence rate of $16.7 \%, 15 \%$, and $10 \%$, respectively. While for women, it was revealed that breast cancer had the highest prevalence $(25.2 \%)$, followed by colorectal (9.2\%) and lung cancer $(8.8 \%)$ ) (World cancer research fund, 2012).

There are clear diversity and multiple ethnicities as well as different religious groups in Iraq, which play a critical role in people's life especially health aspect. Iraqi cancer registry shows that lung cancer is the most frequent cancer among males $(17.2 \%)$, followed by lymphoma $(9.4 \%)$, leukaemia $(9.3 \%)$, bladder cancer $(8.6 \%)$, and gastric cancer $(5.2 \%)$. While for females, breast cancer is the most prevalent cancer $(21.9 \%)$, followed by leukaemia $(8.4 \%)$, lymphoma and myeloma $(7.1 \%)$, lung cancer $(6.2 \%)$, and colorectal cancer (4.9\%). Similar figures are found in Arabic countries with the same modifiable factors as along with poverty and lack of appropriate healthcare services. Generally, data concerning cancer are scares in Arab world and only few studies in this regard have been done. According to World Health Organization (WHO), annual cancer mortality is going to rise from 7.6 million 
in 2008 to around 13 million in 2030 (Ismail et al., 2013).

In health behavioural theories, risk is not solely affected by cognition, but other factor such as favourable attitude are linked with low risk perception (Kelly et al., 2013). Cancer perception plays a crucial role in motivating people to adopt healthy behaviour. Chronic disease susceptibility is affected by modifiable risk factors such as low physical activity, unhealthy diet, smoking, and alcohol drinking. Hence, understanding individual behaviour and discovering factors affecting an individual's decision are critical. Moreover, environmental and social characteristics surrounding people play decisive roles in individual's health decision. Thus, immediate exposure to disease may result in increase of vulnerability to it, enhancing disease risk perception (Schmiedebergs and Pharmacol, 2017). Most of chronic diseases are subtle such as hypertension and cancer, thus majority of affected people do not know about and there will not be any further investigation and medical exam unless the illness is established. People in this stage perceive themselves as healthy individuals (Salman, 2012). Perception of cancer might motive individuals to do screening and lead them to do less risky health behaviour. Those with underestimation of cancer risk perception are less likely to undergo health screening, while others with overestimation of cancer risk perception incline to do health screening and other health activities in order to minimise their worries and put burden on health system (Kim et al., 2014). According to American Cancer Society, more than half of males and about one third of females are affected by cancer . Primary and secondary preventions would prevent and early detect sorts of cancers such as lung and cervical one. It might also lessen the effect of the tumour if detected early and makes course of the treatment shorter. Thus, Perception of cancer risk and severity would affect individual thinking and behaviour and ultimately moves towards healthy preventive behaviour (Werk et al., 2017). The aim of this study was to explore perceived susceptibility, severity and benefit of screening towards cancer among Iraqi adults living in Baghdad.

\section{Materials and Methods}

This cross-sectional study was conducted in Baghdad from June 2016 to October 2016. Our inclusion criteria were defined as follows: aged between 18 to 40 years and not being diagnosed with any chronic diseases. The study was first piloted and validated (Harith and Shamsul, 2017). Sample size was calculated using Fleiss formula (Fleiss, 1981) and difference in proportion in level of education among gender (Al Shafaee et al., 2008).

Participants were selected using multi-stage sampling method. Firstly, all districts and sub-districts were selected through cluster sampling, and then a certain number of clusters were selected as primary sampling unit (PSU). Participants, as the secondary sampling unit (SSU), were chosen conveniently from these clusters. In total, 700 participants were recruited according to our inclusion criteria.
To best of our knowledge, no study has been conducted in this field in Iraq

A self-administered questionnaire was distributed among the participants. The questionnaire was adopted and adapted from that presented by Hafiza et al., (2013) and Figueiras and Alvis (2007). Forward- and Back-translations were conducted by Al-Hikma Institute for Legal Translation.

The questionnaire consisted of the two following sections: sociodemographic section that included information about education level, working status family history of cancer, smoking, alcohol consumption, marital status, BMI, and the amount of exercise done by the participants.

The second section was related to perceived susceptibility, severity and benefits of screening, which were rated by using 5 -point Likert scale $(1=$ strongly disagree, $2=$ disagree, $3=$ not sure, $4=$ agree, $5=$ strongly agree). This includes: health preventive behaviour (practice prayer, using herbal and traditional medicine, do regular follow up and screening, search for nutritional information), health practice and finding health information ( while surfing the internet, from television programs, from health personnel, or family relatives or friends) Questions asked were concerning perception of severity of disease, probability of disease and perception of perceived benefits of screening disease from screening their perceived susceptibility to having the disease, perceived benefits of screening, treatment control (whether disease can be prevented before its happening, treatment can control the disease, treatment will be effective in curing the illness), personal control (course of the disease depends on the person, illness might get better or worse depending on the person), timeline ( the disease will last for long time, it will be permanent, symptoms will comes off and on, it will occurs in cycles), consequences ( the disease has serious financial consequences, the disease has serious burden on the family members), coherence ( the person does not understand the illness, the symptoms of the cancer are puzzling to the person), emotional ( the person get upset when thinking about it, get depressed, get feeling of afraid, becomes anxious).

All the participants were informed about the objectives of the study and written informed consent was obtained from each of the participant.

This study was approved by National University of Malaysia Medical Centre (ethical code: FF-2016-261).

\section{Results}

As shown in Table 1, most of the participants were males $(n=456,65.1 \%)$. In terms of marital status, it was shown that $437(62.4 \%)$ of the participants were married. In total, we found that the number of participants who had high education $(506,72.3 \%)$, were employed (503, $71.9 \%$ ), had abnormal BMI (450, 64.3\%), had low physical activity $(460,65.7 \%)$, and had no experience of smoking $(576,82.3 \%)$ or alcohol drink $(655,93 \%)$ were higher. Regarding family history of cancer, $59(8.4 \%)$ had positive history and $641(91.9 \%)$ had negative history.

Around $35 \%$ of our participants got their health 
Table 1. Sociodemographic Characteristics

\begin{tabular}{|c|c|c|}
\hline Factors & Frequency & Percentage \\
\hline \multicolumn{3}{|l|}{ Gender } \\
\hline Male & 456 & 65.1 \\
\hline Female & 244 & 34.9 \\
\hline \multicolumn{3}{|l|}{ Marital status } \\
\hline Married & 437 & 62.4 \\
\hline Others & 263 & 37.6 \\
\hline \multicolumn{3}{|l|}{ Education } \\
\hline Low & 194 & 27.7 \\
\hline High & 506 & 72.3 \\
\hline \multicolumn{3}{|l|}{ Employment } \\
\hline Yes & 503 & 71.9 \\
\hline No & 197 & 28.1 \\
\hline \multicolumn{3}{|l|}{ BMI } \\
\hline Abnormal & 450 & 64.3 \\
\hline Normal & 250 & 35.7 \\
\hline \multicolumn{3}{|l|}{ Exercise } \\
\hline low activity & 460 & 65.7 \\
\hline active & 240 & 34.3 \\
\hline \multicolumn{3}{|l|}{ Smoking } \\
\hline Yes & 124 & 17.7 \\
\hline No & 576 & 82.3 \\
\hline \multicolumn{3}{|l|}{ Alcohol } \\
\hline Yes & 45 & 6.4 \\
\hline No & 655 & 93.6 \\
\hline \multicolumn{3}{|l|}{ Family history } \\
\hline Yes & 59 & 8.4 \\
\hline No & 641 & 91.9 \\
\hline
\end{tabular}

information while surfing on the internet, and around $40 \%$ resorted to the magazines, $25 \%$ got their information from TV or radio programs, and 40\% obtained their health information from medical staff and their own family members, relatives, and friends.

More than $50 \%$ of the participants resorted to du'a and prayers. Only around $18 \%$ used herbs as some sort of complementary medicine and did screening procedures. In addition, approximately $44 \%$ used nutritional health , and only $11 \%$ practiced some forms of exercises.

Table 2 shows that majority of participants had low perception (62.4\%), while only 263 had good perception $(37.6 \%)$. In terms of perceived severity of cancer, around $418(59.8 \%)$ participants had low perceived threat, while around $40.2 \%$ had good perceived severity. On the other hand, the results showed that participants had

Table 2. Perceived Susceptibility, Severity and Benefits of Screening

\begin{tabular}{lcc}
\hline & High & Low \\
& Frequency (\%) & Frequency (\%) \\
\hline Perceived Susceptibility & $263(37.6 \%)$ & $437(62.4 \%)$ \\
Perceived Severity & $282(40.2 \%)$ & $418(59.8 \%)$ \\
Benefits of Screening & $396(56.6 \%)$ & $304(43.4 \%)$ \\
\hline
\end{tabular}

Table 3. Perceived Susceptibility to Cancer by Sociodemographic and Domains, Hierarchal Linear Regression

\begin{tabular}{lccc}
\hline variable & $\mathrm{B}$ & $\mathrm{SE}$ & $\beta$ \\
\hline Constant & 18.49 & 2.58 & \\
Gender & 0.33 & 0.36 & $0.03^{*}$ \\
Marital status & 0.45 & 0.48 & 0.01 \\
Education & 0.3 & 0.33 & 0.04 \\
Employment & 0.09 & 0.02 & 0.01 \\
Smoking & 0.18 & 0.49 & 0.04 \\
Alcohol use & 0.17 & 0.77 & 0.06 \\
Finding info & 0.01 & 0.05 & 0.02 \\
Prevention & 0.14 & 0.05 & $0.11^{*}$ \\
Behaviour & 0.13 & 0.05 & $0.06^{*}$ \\
Practice & 0.06 & 0.04 & 0.52 \\
Treatment & 0.07 & 0.06 & $0.04^{*}$ \\
Personal & 0.39 & 0.13 & 0.19 \\
Timeline & 0.14 & 0.06 & $0.08^{*}$ \\
Consequence & 0.11 & 0.14 & 0.03 \\
Coherence & 0.16 & 0.1 & 0.06 \\
Emotional & 0.1 & 0.06 & $0.07^{*}$ \\
$\mathrm{R}^{2}$ & 0.059 & & \\
F & $2.862^{*}$ & & \\
$\Delta$ R & 0.33 & & \\
$\Delta$ F & 4.025 & & \\
\hline
\end{tabular}

Hierarchal linear regression test, two-sided $\mathrm{p}$ value $<0.05$, model 3 reported

better perception to the benefits of screening where more than half (56.6\%) had good one, while $43.4 \%$ had low perception to screening benefits .

Hierarchal linear regression test was conducted for the factors, and model 3 revealed that the results were statistically significant between perception domains of treatment control, personal control, timeline, consequences, and coherence and emotional with the outcome . It was shown that $\mathrm{R}^{2}=0.059, \mathrm{~F}=2.862$, and p-value of model 3 was below 0.001 . We found that our participants had some degree of worry, anxiety, and knowledge over the disease course compared to others .

\section{Discussion}

This study examined cancer perceived susceptibility and severity as well as perceived benefits of screening among young Iraqis living in Baghdad. The explanatory model explained around $38 \%$ of the variance in the final model in this investigation, which is vital in explanation of the risk to cancer perception.

Cancer perception level was generally low among our participants regardless of their level of education, of whom $72 \%$ had tertiary level education.

Our finding was consistent with other studies indicating that perceived susceptibility to cancer and prevention behaviour play an important role in chronic disease prevention (Lee et al., 2010). This study emphasized on 
healthy people and their perspective thinking regarding cancer, how their cancer risk perception and diseaserelated evolves. It was revealed that demographic factors like gender, marital status, and level of education were found to play a role in cancer risk perception. similarly, Malmusi et al. yielded that women had lower level of cancer risk perception than men (Malmusi et al., 2012). Castillo et al., also found significant difference between men and women in this regard. However, Barreto and de Figueiredo observed no difference between men and women in terms of the level of cancer risk perception (Barreto and de Figueiredo, 2009).

Considering the effect of education level on cancer risk perception level, our finding was similar with that of other studies (Olszanecka-Glinianowicz and Chudek, 2013; Dieng et al., 2014). Overall, these sociodemographic findings were found to be minimal to the statistical model possibly due to the fact that perception itself is a complex structure and varies from one situation to another and across different individuals. the way through which individuals perceive health problems and make decision concerning their own behaviour do not always follow a logical pattern (García et al. 2005). Furthermore, cancer preventive behaviours and health seeking information were positively correlated with outcome. More than $70 \%$ of our participants obtained their health information from TV programs and more than $80 \%$ from medical personnel, exemplifying that health seeking information is crucial and a step towards improving behaviour to a healthy one, as it has been known that early detection is highly associated with enhanced disease outcome. In similar vein, Ahmad et al., (2014) reported that about one fourth of participants $(n=950)$ were engaged in healthy seeking attitude for cancer prevention and early detection. Additionally, another study showed that these participants had significant differences in terms of treatment, timeline, and emotional domains, may indicating that they perceived the illness to get longer course of treatment, will continue for longer time and drastically worry and fear from it. This is consistent with other studies (Hopman and Rijken, 2015). Individuals even with high level of education have variant understanding and explanations when it comes to illness perception possibly because they do not have enough information concerning the cancer. Psychological factors and health behaviour are both recognised factors and hence play a vital role in perceived health (Freidoony et al., 2015).

There is emphasis that people who positively engaged with screening also have few barriers . The relationship between emotional domain and health engagement in screening behaviour was negative. The more emotional distress was associated with less likely engagement.

It was found that around $50 \%$ of these cancers could be prevented if people had adequate cancer risk knowledge that is satisfactory used to reduce the prevalence. Perceived susceptibility to cancer is a complex process and interaction between different systems which bring about the health behaviour decision according to the behavioural theories. Thus, understanding people's perception to cancer will be of great benefit to improve cancer risk perception and make proper health management programs
(García et al., 2005). Additionally, health behaviour is dependent on health belief as a determinant from which the health theories are developed. It was demonstrated that people would engage in favourable health behaviour if they perceived themselves at risk of a health condition (Wilkinson et al., 2009). Moreover, perception of health people is different from those who are diseased. In other words, healthy individuals perceived cancer as less threatening and less severe than cancerous patients. On the other hand, patients suffering from cancer had a more realistic perception to cancer than healthy individuals. On the other hand, those who did care for ill patients did not develop the same feelings as their patients (Castillo et al., 2011) .

Previous literature found that cancer screening was the sole mode of preventive behaviours among Iraqi, especially for breast and lung cancers (Alwan, 2010; Habib et al., 2016; Sheet et al.. 2012). When people believe that the health benefits that they receive are superior to any other unwanted side effect from the procedures. Action only started when people feel they are at risk, thus they engage themselves in risk-reducing behaviours or get involved in screening procedures (Godino et al., 2014).

Thus, prevention plays an important role. In order to develop a proper and successful preventive program, knowledge and perception of the cancer should be increased. Moreover, attitude towards screening procedures should be positive to enable the satisfactory process .

In conclusion, this study was the first to investigate the perceived susceptibility to cancer among healthy young adult living in Baghdad. Perceived susceptibility to cancer with its inclusion of psychological factors and behaviour are important contributors to self-perceived health. Hence, there is association between perception and future morbidity and mortality, thus it is crucial for public health policy. Comprehensive health programs that include health promotion campaigns and proper health care services are warranted to deal with primary prevention .

\section{Acknowledgements}

\section{Strength and limitations}

The main strength of this study is that it examined a specific belief of healthy young individuals toward cancer. In addition, it shed the light towards an important public health issue and warranted more research in this field because perceived susceptibility to illness reflects the health behaviour and future coping of people with their illness. Regarding limitation, it might be the study design which is cross sectional study in which no causal relationship is determined, although this study was conducted to explore the perception towards cancer rather than determine causality.

\section{References}

Ahmad MM, Dardas L, Dardas L, Ahmad H (2014). Colorectal cancer in Jordan: prevention and care. Glob Health Promot, 


$$
\text { 0, 1-9. }
$$

Al Shafaee MA, Al-Shukaili S, Rizvi SG, et al (2008). Knowledge and perceptions of diabetes in a semi-urban Omani population. BMC Public Health, 8, 249.

Barreto SM, de Figueiredo RC (2009). Chronic diseases, self-perceived health status and health risk behaviors: gender differences. Rev Saude Publica, 43, 38-47.

Brewer NT, Weinstein ND, Cuite CL, Med AB (2004). Risk perceptions and their relation to risk behavior. Ann Behav Med, 27, 125-30.

Castillo A Del, Godoy-Izquierdo D, Vázquez ML, Godoy JF (2013). Illness beliefs about hypertension among non-patients and healthy relatives of patients. Health (Irvine Calif), 5, 47-58.

Dieng M, Watts CG, Kasparian NA, et al (2014). Improving subjective perception of personal cancer risk: Systematic review and meta-analysis of educational interventions for people with cancer or at high risk of cancer. Psychooncology, 23, 613-25.

Eldeek B, Alahmadi J, Al-Attas M, et al (2016). Knowledge, perception, and attitudes about cancer and its treatment among healthy relatives of cancer patients: single institution hospital-based study in Saudi Arabia. J Cancer Educ, 29, 772-80.

Ewaid SH, Al-Azzawi LHA (2016). Breast cancer risk assessment by Gail Model in women of Baghdad. Alexandria $J \mathrm{Med}, \mathbf{5 3}, 3$.

Figueiras MJ, Alves NC (2007). Lay perceptions of serious illnesses: An adapted version of the Revised Illness Perception Questionnaire (IPQ-R) for healthy people. Psychol Health, 22, 143-58.

Fleiss JL (1981). Statistical methods for rates and proportions. New York: John Wiley and Sons, 2, pp139.

García M, Fernández E, Borràs JM, et al (2005). Cancer risk perceptions in an urban Mediterranean population. Int $J$ Cancer, 117, 132-6.

Godino JG, van Sluijs EMF, Sutton S, Griffin SJ (2014). Understanding perceived risk of type 2 diabetes in healthy middle-aged adults: A cross-sectional study of associations with modelled risk, clinical risk factors, and psychological factors. Diabetes Res Clin Pract, 106, 412-9.

Habib O, Al-Asadi J, Hussien O (2016). Lung cancer in Basrah, Iraq during 2005-2012. Incidence and time trend. Saudi Med J, 37, 1214-9.

Hafizah P, Zaleha MI, Shamsul AS (2013). The validation of questionnaire on risk perception of developing five most common non-communicable diseases in Malaysia. $J$ Nurs Health Sci, 1, 29-35.

Harith A, Shamsul S (2018). Perceived susceptibility to chronic disease among healthy people questionnaire: dev. Malaysian $J$ Public Health Med, 18, 8-9.

JafarTH, Haaland BA, RahmanA, et al (2013). Non-communicable diseases and injuries in Pakistan: Strategic priorities. Lancet, 381, 2281-90.

Kim JH, Park EC, Yoo KB (2014). Impact of perceived cancer risk on the cancer screening rate in the general Korean population: Results from the Korean health panel survey data. Asian Pac J Cancer Prev, 15, 10525-9.

Lee JE, Han HR, Song H, et al (2010). Correlates of self-care behaviors for managing hypertension among Korean Americans: A questionnaire survey. Int J Nurs Stud, 47, 411-7.

Malmusi D, Artazcoz L, Benach J, Borrell C (2012). Perception or real illness? How chronic conditions contribute to gender inequalities in self-rated health. Eur J Public Health, 22, 781-6.

Metcalfe KA, Quan ML, Eisen A, et al (2013). The impact of having a sister diagnosed with breast cancer on cancer-related distress and breast cancer risk perception. Cancer, 119, 1722-8.

Olszanecka-Glinianowicz M, Chudek J (2013). The level of health education in the Polish population. Ann Agric Environ Med, 20, 559-65.

Salman KF (2012). Health beliefs and practices related to cancer screening among Arab Muslim women in an urban community. Heal Care Women Int, 33, 45-74.

Schmiedebergs N, Pharmacol A (2017). HHS public access. $\mathbf{5 5}, 1-23$.

Sheet SY, Sheikha AK, Saeed AM, et al (2012). Colorectal cancer: Is the incidence rising in young Iraqi patients?. Asia Pac J Clin Oncol, 8, 380-1.

Torre LA, Bray F, Siegel RL, et al (2015). Global cancer statistics. CA Cancer J Clin, 65, 87-108.

Werk RS, Hill JC, Graber JA (2017). Impact of knowledge, self-efficacy, and perceived importance on steps taken toward cancer prevention among college men and women. J Cancer Educ, 32, 148-54.

Wilkinson AV, Vasudevan V, Honn SE, Spitz MR, Chamberlain RM (2009). Sociodemographic characteristics, health beliefs, and the accuracy of cancer knowledge. $J$ Cancer Educ, 24, 58-64.

\section{(ब) $(1)$}

This work is licensed under a Creative Commons AttributionNon Commercial 4.0 International License. 